\title{
Fremdsprachengeragogik: Motivationen älterer Fremdsprachenlerner
}

\author{
Annette Berndt
}

1. Warum ältere Menschen Fremdsprachen lernen: Eine empirische Erhebung Die Bevölkerungsstruktur der Industrieländer ist im Wandel begriffen: Demographischen Berechnungen zufolge wird die Zahl der Menschen über 60 Jahre in den kommenden 30 Jahren europaweit auf ca. $30 \%$ der Gesamtbevölkerung ansteigen, auf Deutschland bezogen geht man von über 35\% aus (Wingen / Cornelius 1994: 8). Die stetig wachsende Zahl älterer Menschen tritt infolgedessen immer stärker ins politisch-gesellschaftliche Bewußtsein der einzelnen Länder. Der Rat der Europäischen Union reagiert auf diese Situation, die durchaus auch schwerwiegende finanzpolitische Folgen haben wird, mit Ratschlägen zu einer »Politik des lebenslangen Lernens«, bei der das Lernen älterer Menschen in Beziehung zum Arbeitsmarkt stehen sollte (vgl. Klercq 2000: $143 \mathrm{f}$.). Allerdings handelt es sich hierbei um eine Zukunftsvision, denn betrachtet man die Bildungsangebote für ältere Menschen heute, so liegen die Schwerpunkte weniger auf einer berufsbezogenen Weiterbildung, sondern vielmehr auf einem breiten Angebot sinnvoller Freizeitbeschäftigung. Europaweit haben sich inzwischen Institutionen etabliert, die älteren Menschen die Möglichkeit zur Bildung geben. In Deutschland sind die Volkshochschulen der größte öffentliche Anbieter von Bildungsveranstaltungen für ältere Menschen; Fremdsprachenkurse sind dabei integraler Bestandteil des geragogischen Angebotes. Für das Jahr 1997 verzeichnet die Statistik der Volkshochschulen in allen Sprachkursen deutschlandweit 20\% Teilnehmer zwischen 50 und 64 Jahren und ca. 7\% Teilnehmer, die über 65 sind (Deutsches Institut für Erwachsenenbildung 1999: 52 f.).

In meinem Grundsatzartikel zur Fremdsprachengeragogik (Info DaF 1997, 69-77) hatte ich bereits auf die Relevanz dieser neuen Zielgruppe für die Fremdsprachendidaktik aufmerksam gemacht. In der Zwischenzeit ist über die Auswertung qualitativer Interviews ein genaueres Profil der älteren Fremdsprachenlerner von heute entstanden. Befragt wurden Deutschlerner einer Seniorenuniversität in Rom und einige Fremdsprachenlehrer, die ältere Menschen in Deutsch als Fremdsprache dort unterrichten. Die Seniorenuniversitäten, die sog. »Università della Terza Ėtà«, die in allen größeren Städten Italiens existieren, bieten ähnlich den deutschen Volkshochschulen ein reichhaltiges Bildungsangebot speziell für Senioren an.

Die befragten Senioren besuchen Kurse in Deutsch als Fremdsprache zum Teil seit mehreren Jahren und auf drei verschiedenen Kursniveaus. Die folgenden Ausführungen werden mit kurzen Ausschnitten aus transkribierten und ins Deutsche übersetzten Interviews illustriert. Die Gültigkeit der Aussagen italienischer Senioren auch für deutsche Fremdsprachenlerner im Alter ist inso- 
fern gegeben, als die geragogische Literatur in allgemeinen Untersuchungen zum Bildungsverhalten älterer Deutscher zu ganz ähnlichen Ergebnissen kommt (vgl. Kaiser 1997).

\section{Einen alten Traum realisieren}

Bildung im Alter trägt häufig kompensatorische Züge:

"Nachdem ich mich habe frühpensionieren lassen, wollte ich endlich mal machen, was mich immer interessiert hat, was ich aber nie habe machen können. Früher war ich ja im technischen Bereich; da habe ich mich überhaupt nicht um Literatur oder Sprachen gekümmert. Dann bin ich an die Seniorenuniversität gekommen, um das Verpaßte nachzuholen.« (Marco)

»Ich wollte immer Sprachen studieren - in der Schule, als Kind habe ich nur Französisch gelernt. So habe ich mir jetzt den Traum erfüllt, Sprachen zu lernen und ins Ausland zu fahren. Das ist eine Sache, die mir sehr viel Spaß macht.« (Anna)

Einer der sehr häufig genannten Gründe, einen Fremdsprachenkurs im Alter zu besuchen und für Weiterbildung in späteren Lebensphasen überhaupt, ist der Wunsch, Bildung nachzuholen, die in früheren Lebensphasen nicht möglich war.

Einer Untersuchung $\mathrm{zu} »$ Zielen und Träumen für den Ruhestand « von 5065jährigen Menschen zufolge (Freericks 1990) stehen Bildung und Sprachenlernen an dritter Stelle (ca. 40\% der Befragten) nach Reisen und Hobby. Die Kompensation entgangener Bildungschancen durch Sprachenlernen im Alter kann ohne weiteres auch als ein Beitrag zur gerechteren Verteilung von Bildungschancen aufgefaßt werden, zieht man in Betracht, daß die Bildungschancen im Jugendalter der heute 60jährigen im Vergleich zur heutigen Jugend ungleich schlechter waren. Dieses Faktum »defizitärer Bildungsbiografien « bezieht sich besonders auf die heute älteren Frauen (vgl. Kaiser 1997: 33). Bei einem Ge- schlechtervergleich »schälen sich besonders die 60-65jährigen Frauen als saktive neue Alte heraus. Im Vergleich mit gleichaltrigen Männern und den 7075jährigen beiderlei Geschlechts werden für diese Gruppe die größten Anteile an Sport- und Reiseaktivitäten sowie Teilnahmen an Bildungsveranstaltungen angegeben « (Karl 1996: 37). Diese offenbar höhere Aktivitätsbereitschaft von Frauen im Alter ist neben der allgemeinen zahlenmäßigen Überlegenheit im Alter einer der Gründe für den hohen Frauenanteil (ca. 80\%) in Bildungsveranstaltungen und speziell auch in Fremdsprachenkursen für Ältere. Gerontologie und Geragogik sprechen in diesem Zusammenhang von einer »Feminisierung « des Alters (vgl. Sagebiel 2000).

\section{Aufrechterhalten von Kontinuität}

Viele ältere Menschen, die Fremdsprachenkurse besuchen, haben sich während ihres gesamten Lebens mit dem Thema Sprachenlernen mehr oder weniger intensiv beschäftigt. Auch Kaiser (1997: 91) bestätigt dieses Phänomen der Kontinuität von Bildung allgemein durch das Leben bis ins hohe Alter in ihrer empirischen Studie zum Seniorenstudium. Allerdings ändern sich im Alter die Kontexte, in denen die Fremdsprachenkenntnisse angewendet werden können. Oftmals fallen wesentliche Referenzpunkte wie zum Beispiel der Beruf weg: Eine unweigerliche Folge dessen wäre daher ein Verfall der Sprachkompetenz. Um dem vorzubeugen und um das Sprachwissen zumindest auf dem gewohnten Niveau zu halten, wenn nicht zu verbessern, wird der Kontakt zu einem Sprachkurs gesucht:

»Ich wollte einfach sehen, an wie viel ich mich noch erinnere. Früher habe ich mal mit der Sprache gearbeitet und sie angewendet, dann längere Zeit nicht mehr. Das Anwenden schützt vor dem Vergessen. Ich spreche ja auch noch Französisch und ein 
bißchen Spanisch, das habe ich vor einiger Zeit hier an der Seniorenuniversität begonnen.«(Lara)

Ältere Menschen sind häufig Wiederlerner einer Sprache; der Unterricht hat somit auch die Aufgabe einer Reaktivierung von Sprachkenntnissen. Die häufig anzutreffende differenzierte Mehrsprachigkeit kann für die aktuelle Fremdsprache in vielfacher Weise genutzt werden, indem zum Beispiel auf ein bereits vorhandenes Strategiewissen zurückgegriffen werden kann und beispielsweise auch lexikalische Transfers aus genetisch verwandten Sprachen vollzogen werden können (vgl. Mißler 1999). Fremde Sprachen zu sprechen, gehört für diese älteren Menschen zu den Fertigkeiten, die sie ein Leben lang gepflegt haben und deren Weiterbeherrschen die Aufrechterhaltung eines Teils ihrer Identität bedeutet. Hierzu kann auch gehören, daß man sich mit Gleichaltrigen mißt - ein Ansinnen, das normalerweise nur jüngeren Menschen zugeordnet würde:

»Ich kann mich in der Klasse mit anderen konfrontieren, ja, das ist mir sehr wichtig, (lacht), vielleicht bin ich zu pessimistisch (lacht) (Pause), denn als ich noch arbeitete, war ich ja aktiv, da hatte ich immer den Vergleich mit den anderen. Ich glaube, daß es wichtig ist, einen Vergleichspunkt zu haben, um sich selbst einschätzen zu können. Als wir zum Beispiel nach München fuhren, mußte ich mir eingestehen, daß ich kaum etwas verstand, das hat meine Selbsteinschätzung im Deutschen korrigiert.« (Cornelia)

Indem sich Cornelia, ehemals Lehrerin, mit Gleichaltrigen in einem Klassenverband konfrontiert, erhält sie über die Lernkontinuität hinaus Informationen über ihren Leistungsstand im Vergleich zu den anderen. Der Kurs wird somit zu einem wichtigen Punkt im Koordinatensystem ihrer Selbsteinschätzung. Der Wegfall des beruflichen Kontextes, der besonders bei sog. erfolgreichen Men- schen auch Konkurrenzsituationen mit sich bringt, wird durch den Gruppenkontext ersetzt und entsprechend auch mit Anforderungen besetzt. Der Kurs kann insofern zum Erfolgsmesser werden; der ältere Mensch versucht über sein Gelingen oder Mißlingen im Unterricht Informationen zu bekommen, wie er leistungsmäßig in der Kontinuität seines Lebens abschneidet und zudem im Vergleich zu den Gleichaltrigen. Auch Anna weist dem Sprachenlernen die Funktion der eigenen Leistungsmessung zu: »Das ist so eine Art Probe meiner selbst geworden, ob ich es nun schaffe oder nicht «.

An diesem Punkt wird die didaktische Kategorie des Erfolges virulent, denn mangelnde oder zu hohe Anforderungen können zu Frustrationen und im Rückkehrschluß zu einer negativen Selbsteinschätzung führen. Kaiser (1997: $172 \mathrm{ff}$.) weist darauf hin, daß das Studium von Älteren Menschen als Bestätigungsraum ihrer eigenen Leistungsfähigkeit dient, indem sie dem Lernen im Alter »eine entwicklungsfördernde Wirkung « zur Steigerung des Selbstbewußtseins konstatiert. Fremdsprachenkurse für ältere Menschen müssen sich also in einem Bereich zwischen Anspruch und Bestätigung bewegen, der in jedem Fall über den bloßen Zeitvertreib hinausweist.

\section{Zeitorganisation und sinnvolle Frei- zeitbeschäftigung}

Zunächst kommt das Einschreiben in einen Fremdsprachenkurs dem Wunsch entgegen, in ein festes zeitliches Raster eingebunden zu sein, denn die »neu gewonnene Freiheit« (Berger/Hradil 1994) durch das Ausscheiden aus dem Berufsleben - im Fachjargon »Entberuflichung « genannt - oder die Tatsache, daß erwachsene Kinder das Haus verlassen haben (empty-nest-syndrom), stellt ältere Menschen plötzlich vor die Tatsache, die freie Zeit selbst einteilen zu müssen. Gewohnt, 
daß die verfügbare Zeit großenteils von außen durch Berufstätigkeit oder Familie mitbestimmt war, müssen nun Möglichkeiten gefunden werden, die freie Zeit sinnvoll zu gestalten. Prahl/Schroeter schreiben hierzu:

»Die >späte Freiheit ‘ hat zwei Gesichter: Sie erscheint zum einen als Befreiung von Pflichten und Zwängen, doch sie läßt auch den Wunsch nach Struktur und sinnvollem Handeln wach werden«. (Prahl/Schroeter 1996: 147)

Das Alter als postfamiliäre und nachberufliche Lebensphase führt zu dem Anspruch einer selbständigen Zeiteinteilung, wobei regelmäßige Besuche von Volkshochschulkursen beispielweise wichtige Fixpunkte der zeitlichen Tagesund Wochenstrukturierung bilden können. Das Alter wird »vom Ruhestand zur individuellen Herausforderung " (Prahl/ Schroeter 1996: 42) und Weiterbildung im Alter kann helfen, diesem Anspruch gerecht zu werden. Nicht umsonst ist die Weiterbildung im Sinne von Weiterlernen eine der Grundfesten des Konzeptes zum »erfolgreichen Altern «(Baltes/Kohli/Sames 1989). Das Sprachenlernen kann somit den Stellenwert eines sinnvollen Zeitvertreibs einnehmen:

»Als meine Mutter noch lebte, hatte ich ja nichts anderes zu tun, als mit ihr Bridge zu spielen. Dann, als auch sie gestorben war, wusste ich nichts mehr anzufangen mit mir und bin dann eben zur Seniorenuniversität gegangen. [...] Mit Deutsch habe ich dann angefangen, weil ich endlich etwas machen wollte, das mir Mühe abverlangte.« (Olga)

Kaiser schreibt in diesem Sinne:

»Zur befriedigenden Gestaltung der nachberuflichen/nachfamiliären Phase reicht es nicht, beliebigen Freizeitbeschäftigungen nachzugehen, mit denen die Zeit mehr oder weniger stotgeschlagen « wird; vielmehr ist dazu erforderlich, eine Aufgabe zu finden, die dem/der einzelnen Sinn vermittelt und als lebensbereichernd erfahren wird «. (Kaiser 1997: 151)
Allerdings wird dem Aspekt der Bildung nicht immer nur die Funktion einer Freizeitbeschäftigung zugewiesen. Emma beispielsweise macht als ehemalige Universitätsprofessorin auf die Lebensfunktion von Bildung aufmerksam, deren Fehlen auch im Alter politische Auswirkungen haben kann:

»Es kommt sehr stark auf das Bildungsniveau der Menschen an. Im englischen Sinne, als education, die erlaubt, die Vorurteile und die Barrieren $\mathrm{zu}$ überwinden. Auch die älteren, wenn sie nicht genügend gebildet sind in diesem Sinne, sind eher ein Hindernis, als daß sie auf dem Wege der [europäischen, die Autorin] Vereinigung helfen würden. [...] Und ich muß ehrlich sein: Der Großteil der Leute in meinem Alter denkt überhaupt nicht nach. [...] Man muß endlich kapieren, daß die Bildung nicht nur dazu da ist, eine Arbeit zu finden, sondern daß sie helfen muß zu leben.« (Emma)

Jedoch stehen nicht alle befragten Senioren unter der Notwendigkeit, freie Zeit über Kursbesuche strukturieren zu müssen. Irene, die in den Deutschkurs kommt, da ihr Sohn eine deutsche Frau geheiratet hat, entwirft ein ganz anderes Bild zeitlicher Organisation:

»Die Kinder MÜSSEN ja in die Schule. Bei uns ist das so, daß wir selbst verantwortlich sind, ob wir etwas tun oder nicht. Die Schulkinder haben ja sonst nichts zu tun, aber wir in unserem Alter sind Ehefrau, Mutter, Oma, Uroma, Haushälterin, Gärtnerin, Putzfrau; alles dreht sich um dich und du mußt funktionieren, man kann ja nicht snein sagen. Darf eine Oma zum Enkelkind snein s sagen? Und jetzt ist mein Mann auch noch ziemlich krank, wir sind dauernd unterwegs zu irgendwelchen Ärzten. Wie soll ich das alles schaffen? Und da bleibt das Lernen immer an letzter Stelle.« (Irene)

Irene beschreibt ihr Eingebundensein in eine Mehr-Generationen-Familie als starke Belastung, die sie vom Lernen der deutschen Sprache oft abhält. Allerdings ist das Modell der Mehr-Generationen- 
Familie in allen Industriestaaten in der Abnahme begriffen; ältere Menschen ziehen es immer mehr vor, selbständig zu leben und ihren eigenen Interessen nachzugehen (Lehr 1991: $251 \mathrm{ff}$.). Auch der sog. "Rentnerstress « (Prahl/Schroeter 1996: 148 f.) kann Grund dafür sein, daß ältere Menschen oft angeben, keine Zeit $\mathrm{zu}$ haben und infolgedessen unvorbereitet in den Unterricht kommen. Es handelt sich dabei um die Tendenz älterer Menschen, sich - aus welchen Gründen auch immer - mit Aktivitäten zu überlasten und dadurch in Zeitdruck zu geraten. Anna gibt beispielsweise an, zweimal täglich mit dem Bus zur Seniorenuniversität zu kommen, was einer täglichen Fahrtzeit von über vier Stunden entspricht:

"Morgens mache ich Sport, da schwimme ich, spiele Tennis und mache Selbstverteidigung (lacht), dann fahre ich mit dem Bus nach Hause und komme nachmittags wieder, um die anderen Kurse zu besuchen, das sind die Fremdsprachen, Religion, Geschichte habe ich auch schon gemacht.« (Anna)

\section{Soziale Kontakte}

Ein Phänomen, das mit dem Ausscheiden aus dem Berufsleben und der Reduzierung der Familie bzw. dem Alleinleben durch Verwitwung einhergeht, ist eine Veränderung im Bereich der sozialen Kontakte. Während ältere Studien mit der sog. "Pensionierungsproblematik« (Lehr 1991: 217 ff.) die Sicht eines »social breakdown « (Kuypers/Bengston 1973) verbinden, verdeutlichen neuere Studien die Umstrukturierung des Sozialverhaltens in dieser Lebensphase (Lehr 1991: 239 f.). Dabei wird der Stellenwert neuer sozialer Kontakte betont, wie sie beispielsweise durch die Inanspruchnahme von Bildungsangeboten zustande kommen können. Lehrerin Anke beschreibt die Motivation ihrer älteren Studierenden folgendermaßen:
"Ältere kommen in den Deutschkurs, um ein soziales Erlebnis zu wiederholen, das sie vor Jahrzehnten erlebt haben, nämlich den Unterricht in der Klasse. [...] Für die alten Menschen ist es im allgemeinen wichtig, daß sie das Haus verlassen mit dem Ziel eines sinnvollen Treffens mit anderen Menschen, bei dem sie mit anderen etwas zusammen machen.« (Anke)

In den Jahren vor der Pensionierung wird der sich möglicherweise ändernde soziale Kontext und der Stellenwert von sozialen Kontakten in der nachberuflichen Phase von den Betroffenen selbst kaum wahrgenommen. So steht die Option »Kontakte pflegen « bei einer Befragung von Menschen zwischen 50 und 65 Jahren an achter Stelle ihrer Ziele für die nachberufliche Phase; in der Phase der Pensionierung stellt die Studie eine Verschiebung dieser Option auf Platz drei der tatsächlich ausgeführten Aktivitäten fest. Auf Platz eins und zwei der Rangliste stehen Reisen, Ausflüge und Hobby, auf Rang vier Bildung (Freericks 1990: 31).

Um Sprachen zu lernen, mit Menschen zusammen zu sein und um über die fremde Sprache mit Menschen zu kommunizieren, so thematisiert Anna ihre Beweggründe:

»Ich möchte andere Länder, andere Völker kennenlernen. Ich möchte einfach Kontakt mit Menschen haben. Ich studiere die Sprache nicht wegen der Sprache selber, wegen den Vokabeln und der Grammatik, sondern weil sie ein Medium ist, den Rest der Menschheit zu verstehen, die nicht meine Sprache sprechen. [...] Kürzlich waren wir mit der Klasse in München. Das hat in mir den Wunsch verstärkt, weiter Deutsch zu lernen und auch einen richtigen kulturellen Austausch zu machen - sonst bleibt das Lernen ja ein Selbstzweck. Nein, ich möchte wirklich Kontakt zu den älteren Damen und Herren, die Italienisch lernen in München. Dieser Aufenthalt hat mir wirklich das Ausmaß meiner Unwissenheit gezeigt: Daß ich eben nicht in der Lage war, ein Gespräch auf deutsch zu führen. Ja, ein paar 
Worte habe ich schon gewechselt, aber ich möchte mich besser unterhalten können mit den Leuten, die ja so nett zu uns waren und viel mit uns unternommen haben. Da haben wir wirklich einen freundschaftlichen und auch kulturellen Austausch gemacht. Das war so eine Art Osmose von Wissen und auch der Sprache. Die Deutschen haben Italienisch gesprochen und ich habe Deutsch gesprochen, und wir haben uns gegenseitig korrigiert. [...] In der Klasse habe ich nun viele Freunde, auch deswegen möchte ich den Unterricht nicht verlassen. Die Freunde sind sehr wichtig, ich habe mich in die Klasse eingegliedert." (Anna)

»Einsamkeit« und »Isolation « sind Stichworte, die häufig mit dem Alter in Verbindung gebracht werden. Einsamkeit infolge der Desozialisation im Alter im Sinne einer Loslösung von gewohnten sozialen Kontakten wird in der Sozialgerontologie folgendermaßen operationalisiert: Einsamkeit als Funktion von Langeweile ist die Folge einer mangelnden Rhythmisierung der Tages- und Wochenabläufe (Lehr 1991: 280 ff.). Weiterbildung im Alter mit der Funktion einer sinnvollen Beschäftigung und Zeiteinteilung in der Gesellschaft anderer Menschen dürfte diesem Phänomen entgegenwirken.

\section{Reisen}

»Ja, ich habe eben Sprache gerne, und vom Deutschen konnte ich nur ganz wenige Wörter, und da wollte ich ein bißchen mehr wissen. Und ich reise auch sehr gerne, und da möchte ich im Ausland eben nicht dumm dastehen, wenn ich mich überhaupt nicht verständigen kann.« (Nina)

Der Wunsch zu reisen und im Zielland mit Menschen in der Fremdsprache $\mathrm{zu}$ kommunizieren ist ein häufig genanntes Argument für das Sprachenlernen. In der Tat hat sich die Tourismusbranche seit einigen Jahren auf die Zielgruppe älterer Menschen mit sehr differenzierten Angeboten eingestellt. Der Bildungstourismus bietet zudem spezielle Studienreisen für ältere Menschen an, in die Sprachkurse integriert sind oder die das Sprachenlernen im Ausland als Schwerpunkt anbieten. Zitiert sei an dieser Stelle ein Ausschnitt aus dem Angebot »Sprachurlaub für Menschen ab 50 Jahre« der Carl Duisberg Centren aus dem Jahre 2000, das unter dem Stichwort »Master Class« läuft:

»In angenehmer Atmosphäre erlernen Sie die Landessprache bzw. vertiefen vorhandene Kenntnisse. Sie treffen gleichgesinnte Menschen aus der ganzen Welt, die wie Sie neue Kontakte knüpfen und sich gleichzeitig ohne Lernzwang weiterbilden möchten. [...] Auf gemeinsamen Ausflügen und anderen attraktiven Freizeitaktivitäten erschließst sich Ihnen die Kultur des Landes und der Region in ihrer ganzen Vielfalt«. (1)

Die Zielländer sind an erster Stelle England, gefolgt von Italien, Spanien und Frankreich. Bei näherem Hinsehen verweist dieser Text auf einige Charakteristika, die das Sprachenlernen älterer Menschen im Ausland erfolgversprechend gestalten - wobei das Lernen zu einer »Weiterbildung ohne Lernzwang « mutiert und in »angenehmer Atmosphäre " geschieht. Die Menschen, die der Senior dort antreffen wird, werden als »gleichgesinnt « beschrieben, um auf die Möglichkeit positiver sozialer Kontakte zu verweisen. Durch die Kennzeichnung »ab 50 Jahre« wird ausdrücklich auch die Zielgruppe der »jungen Alten « angesprochen - und zudem auf elegante Weise die Notwendigkeit zur begrifflichen Nennung der Senioren umgangen. »Weiterbildung ohne Lernzwang « entspricht der Auffassung Semmelroths (2000: 273), wonach »Lernen auf Reisen niemals das Erreichen eines strikten Lernziels « beinhalten sollte.

Die Forschungsergebnisse zum Seniorentourismus belegen ein stetiges Anwachsen der Reisetätigkeiten Älterer, die im Vergleich zur Gesamtbevölkerung bezo- 
gen auf Deutschland eindeutig höher ist (Amaschus 1996). So haben im Jahre 1997 ca. $70 \%$ der Senioren zwischen 60 und 70 Jahren und ca. $60 \%$ der über 70 jährigen mindestens eine längere Reise unternommen (Gruner \& Jahr 1998), wobei die Reisehäufigkeit mit jeder Kohorte (i. e. Personengruppe bestimmter Jahrgänge) als anwachsend prognostiziert wird. Nach Wallraven (1995) sind die Hauptinteressen der älteren Reisenden Begegnung und Kommunikation sowie die Realisierung von Bildungs- und Kulturinteressen. Das Sprachenlernen auf Reisen wird somit zunehmend an Wichtigkeit gewinnen und ein relevantes Tätigkeitsfeld für Fremdsprachengeragogen darstellen.

\section{Gedächtnistraining}

Cornelia, nach den Unterschieden beim Lernen in jungen Jahren und heute befragt, weist auf die schwächere Funktionstüchtigkeit des Gedächtnisses hin:

»Der Unterschied? Natürlich das Gedächtnis. [...] Ja, früher, da hatte ich ein gutes Gedächtnis, das wird alles immer langsamer und weniger - und das Sprachenlernen hilft mir eben, diesen Prozeß zu verlangsamen. Manche lösen Kreuzworträtsel deswegen, und ich lerne eben Deutsch, das macht mir mehr Spaß.« (Cornelia)

Das Sprachenlernen als mentale Tätigkeit wird dem Lösen von Kreuzworträtseln vorgezogen, da ersteres anspruchsvollere Prozesse der Informationsverarbeitung im Sinne von Lernen erfordert, während letzteres den bloßen Abruf von Informationen verlangt. Ältere Menschen sind sich der Veränderungen in der Informationsverarbeitung, die mit dem Alterungsprozeß verbunden sind, sehr genau bewußt. Im Vergleich zu Veränderungen im Persönlichkeitsbereich und emotionaler Komponenten liegen zum Altern im kognitiven Bereich eine Vielzahl von Untersuchungen vor, so daß die Ergebnisse als relativ gesichert angesehen werden können. Allerdings darf man an dieser Stelle nicht vergessen, daß Altern kein einheitlicher und interindividuell invarianter Prozeß ist. Vielmehr nehmen mit zunehmendem Alter auch die Unterschiede zwischen den Individuen zu. Zudem ist Altern ein multidirektionaler Prozeß, der intra-individuell unterschiedlich ausgeprägt ist; so kann beispielsweise ein geistig äußerst aktiver Mensch massive Anzeichen organischer Alterung zeigen. Die beiden eben umschriebenen Phänomene der inter- und intraindividuellen Varianz sind zwei der Grundmodelle der Psychologie des Lebenslaufs, um Altern zu operationalisieren (vgl. Baltes 1990).

Kognitives Altern wird hauptsächlich durch Veränderungen in der Intelligenz erhoben, wobei man davon ausgeht, daß es sich dabei um kein einheitliches Merkmal, sondern um ein mehrdimensionales Fähigkeitskonzept handelt. Als Beschreibungsmodell setzte sich die Differenzierung in kristalline und fluide Intelligenzkomponenten durch (Cattell 1963). Die fluiden Komponenten umschreiben die kognitiven Grundfunktionen, die eine flexible Aufnahme und Verarbeitung von Informationen ermöglichen. Diese stark tempoabhängigen Funktionen unterliegen einem progredienten Abbau ab ca. dem 40. Lebensjahr. Die kristallinen Funktionen umschreiben übungs- und bildungsabhängige Leistungen wie Sprachwissen oder spezielles Fachwissen, die bis ins hohe Alter konstant bleiben in ihrer Leistungsfähigkeit. So bleibt eine grundlegende kristalline Variable wie zum Beispiel »Reasoning « bis über das 70. Lebensjahrzehnt hinaus konstant, während beispielsweise die psychometrische Messung fluider Funktionen wie »Wortflüssigkeit« bei älteren Menschen im Vergleich zu Individuen im frühen Erwachsenenalter nur ca. die halbe Leistungsfähigkeit feststellt (Oswald 2000: 
114). Auch bezüglich des Gedächtnisses kann man sehen, daß alle Prozesse, die eine starke Verarbeitungskomponente aufweisen, wie das auch beim fremdsprachlichen Lernen der Fall ist, im Alter Defizite aufweisen. So performieren das Arbeitsgedächtnis als Funktion des Kurzzeitgedächtnisses und das episodische Gedächtnis als Funktion des Langzeitgedächtnisses bei älteren Menschen sehr viel schlechter als bei jüngeren: Je älter ein Mensch ist, umso länger braucht er für En- und Dekodierungsvorgänge und verkraftet zudem immer geringere Informationsmengen (Smith/Earles 1996). Die wissenschaftlichen Untersuchungen bestätigen also Cornelias weit verbreitete Hypothese »langsamer und weniger « bezüglich ihrer Gedächtniskapazitäten. Allerdings soll an dieser Stelle darauf hingewiesen werden, daß über einen Vergleich von Längsschnittuntersuchungen eine starke Kohortenabhängigkeit der intellektuellen Leistungsfähigkeit festgestellt wurde: Die heute 60jährigen performieren dabei um einiges besser als beispielsweise dieselbe Altersgruppe vor 20 Jahren. Dieses Phänomen läßt sich mit dem stetig steigenden Bildungsgrad der Bevölkerung erklären, da intellektuelle Leistungsfähigkeit und Bildung korrelierende Faktoren sind.

Interessant ist, daß das Sprachenlernen von älteren Menschen explizit als Übung zur Aufrechterhaltung der geistigen Leistungsfähigkeit instrumentalisiert wird. So äußert sich Olga folgendermaßen:

»Ich übe mein Gedächtnis mit ganz dummen Dingen wie Kreuzworträtsel - die Ärzte sagen ja, das soll gut sein. Das ist Gymnastik für das Gehirn. So ist das Deutschlernen auch eine Art Gymnastik für das Gehirn - deshalb habe ich auch eine schwierige Sprache gewählt, um diesen Effekt der Gymnastik zu haben.« (Olga)

Die Bezeichnung des Deutschen als "schwierige Sprache" ist aufgrund des deutschen Flexionssystems, der Genera und auch der Syntax im Vergleich zur romanischen Sprache Italienisch berechtigt. Die Behauptung, Deutsch gerade deswegen lernen $\mathrm{zu}$ wollen, weil es schwierig ist, begründet sich offenbar in der Überzeugung, über den anspruchsvollen Lernprozeß der deutschen Sprache geistige Komponenten wie das Gedächtnis ganz besonders trainieren zu können. Der interventionistische Auftrag der Geragogik verbunden mit dem Anspruch der Kompetenzerhaltung weisen jegliches Lernen im Alter als Training der geistigen Fähigkeiten aus; Fremdsprachenkurse sollten insofern wie jedes Bildungsangebot für ältere Menschen eine intellektuelle Herausforderung darstellen (Kaiser 1997: 254).

\section{Motivationen zum Deutschlernen ita- lienischer Senioren}

Die italienischen Senioren weisen ein durchwegs positiv gefärbtes Deutschlandbild auf, wobei ein wesentlicher Unterschied zwischen Männern und Frauen bezüglich der Sympathie für Deutschland auffällt. Während Frauen sich hauptsächlich auf ihre Begeisterung für die deutsche Literatur, Philosophie und Musik beziehen, nennen Männer explizit auch politische Beweggründe, die oft auch mit Erfahrungen der Kindheit in der Phase des 2. Weltkriegs in Zusammenhang stehen. Marco, der im Piemont aufgewachsen ist, beschreibt seine Affinität zur deutschen Sprache folgendermaßen:

»Zum Deutschen habe ich eine ganz besondere Verbindung. Als kleines Kind fühlte ich mich sehr angezogen von den deutschen Soldaten. Als der Krieg aufhörte, da war ich neun Jahre alt, aber meine Erinnerungen daran sind sehr lebendig. In meinem Dorf waren die Soldaten der Wehrmacht, die sich allmählich in die Dorfbevölkerung integrierten; wir Kinder gingen immer zu den Soldaten, da gab es Waffen. Irgendwann waren die Deutschen keine 
Freunde mehr, und meine Mutter verbot mir, zu den Deutschen zu gehen. [...] Ich dachte mir, daß das komisch ist, und ließ mich auch nicht groß beeindrucken, ich ging immer wieder hin, weil es mir dort gefiel. Dann, nach Kriegsende, kamen die Propagandafilme der Amerikaner, die die Deutschen als Dummköpfe darstellten und das deutsche Heer in Mißkredit brachten. [...].« (Marco)

Damiano bezieht sich in seinem positiven Deutschlandbild vor allem auf die deutsche Mentalität und die politische Rolle der Deutschen in Europa:

»Und mir gefällt die deutsche Sprache auch, genauso wie mir die deutsche Mentalität gefällt, das Zurückhaltende, ein bißchen so wie das Englische, man nannte mich eigentlich auch eine Zeitlang >der Deutsche`, weil ich so korrekt und zuverlässig bin. Deutschland spielt eine sehr wichtige Rolle in Europa, da ist dann auch die Sprache wichtig. Deutschland hat in seiner Geschichte in ganz Europa wohl die wichtigsten Persönlichkeiten hervorgebracht auf allen Gebieten. So etwas im Unterricht zu hören ist sehr wichtig. Das alles hat mich dazu gebracht, Deutsch zu lernen. [...] Deutschland hat mir während des Krieges gefallen - und auch danach. Die Deutschen sind ein Volk, das ungern Vertrauen faßt zu anderen Völkern und ich denke immer, daß da etwas Geheimnisvolles dahinter ist, etwas, das ich entdekken möchte. Das reizt mich. Auch die Deutschen, die nach Italien kommen, bleiben immer unter sich, die mischen sich nicht so schnell unter uns Italiener.« (Damiano)

Für Nina sind besonders die deutsche Literatur und Musik wichtig:

»Wir hatten zu Hause einen Gedichtband mit deutschen romantischen Gedichten in italienischer Übersetzung. Die habe ich als junges Mädchen gelesen, die gefielen mir so gut. Die deutsche romantische Literatur, die ist so wunderbar, die Italiener schaffen es irgendwie nicht, so schöne Dinge zu schreiben. Auch die Musik, Schumann und die anderen Komponisten. Jedenfalls kann ich jetzt endlich mal Mörike im Original lesen, das machen wir ja auch im Unterricht, das gefällt mir außerordentlich gut.« (Nina)
Emma betont die differenzierten Ausdrucksmöglichkeiten, die die deutsche Sprache eröffnet:

»Das Englische ist ja bequem, da gibt es auch einiges, was mir gefällt, aber es ist keine Sprache, die mich fasziniert. Es ist nicht synthetisch, eher idiomatisch, aber es gefällt mir nicht, es hat keine Feinheiten wie das Deutsche, auch wenn die Konstruktion streng ist im Deutschen, man kann sich sehr genau ausdrücken. Nicht umsonst ist es die Sprache der Philosophie und der Kunst.« (Emma)

\section{Schlußfolgerungen für die Fremd- sprachengeragogik}

Die vorigen Kapitel zeigen ein sehr breites Spektrum an Motivationen, die ältere Menschen zum Besuch eines Fremdsprachenkurses bewegen. Die Bandbreite reicht von der Suche nach sozialen Kontakten in einem sinnvollen Rahmen bis hin zum zielgerichteten Lernen. Aus der mangelnden Bündelung der Interessen über explizite Zielsetzungen des Fremdsprachenkurses resultiert eine Inhomogenität des Kurses, die sich in den unterschiedlichen Motivationen und dann auch Erwartungen der Teilnehmer begründet. Eine zielgruppenspezifische Methodik und Didaktik des Fremdsprachenunterrichts muß dieser Vielfalt gerecht werden. Die Geragogik als Pädagogik für ältere Menschen ist sich dieser Eigenheit bewußt und hat bereits entsprechende Bildungskonzepte entwickelt.

Ludger Veelken, einer der führenden Theoretiker der Geragogik in Deutschland, begründet die Berechtigung einer speziellen Pädagogik für ältere Menschen folgendermaßen:

»Die spezifische Lebenssituation, die sozioemotionalen Bedürfnisse und intellektuellen Fähigkeiten des alten Menschen erfordern spezifische Lernziele und -inhalte, die sich von denen der Andragogik unterscheiden.«(Veelken 1994: 16)

Weiter schreibt Veelken: 
»Die vorrangig zugrundeliegende Theorie ist in erster Linie die Sozialisationstheorie, die die vielseitige Wechselwirkung von Kultur, Gesellschaft und Individuum beschreibt und deren Ziel die Entfaltung menschlicher Identität in einem konkrethistorischen Kontext ist.« (Veelken 1994: 17)

Ziel von Sozialisationsprozessen ist die Entfaltung von Identität im Lebenslauf; die Geragogik spricht insofern von der sog. »tertiären Sozialisation«, die Menschen ab ca. 50 Jahren betrifft und altersgemäße Anforderungen an die Bildungsarbeit stellt.

Der geragogische Bildungsbegriff bewegt sich demnach zwischen intentionaler und nicht intentionaler Bildung (Semmelroth 2000: 272), i. e. zwischen dem Anspruch, zielgerichtete Lehrangebote zur Verfügung zu stellen, und der Verpflichtung, über das soziale Ereignis des Unterrichts Bedürfnisse wie Kontakte zu Gleichgesinnten zu ermöglichen. Ein grundsätzliches Dilemma der Geragogik ist allerdings, daß ältere Menschen explizit zwar keine zielgruppenspezifischen Konzepte wollen und bevorzugt an allgemeinen Angeboten teilnehmen, aber dennoch spezielle Bedürfnisse haben. Dieser Zwiespalt reflektiert sich beispielsweise in der Problematik der Begrifflichkeiten bei allem, was ältere Menschen betrifft: So wird die Pensionierung zur »Entberuflichung « und Seniorenreisen profilieren sich unter dem äußerst positiv besetzten Titel »Master Class«. Die älteren Menschen werden nicht als solche bezeichnet, sollen aber ihren Bedürfnissen entsprechend bedient werden.

Die Bildungsarbeit mit älteren Menschen steht für Nittel (1999: 357 ff.) im Schnittfeld der drei pädagogischen Subdisziplinen Erwachsenenbildung, Freizeitpädagogik und Sozialpädagogik. Freizeit darf hier allerdings nicht als Komplementärbegriff zu Erwerbsarbeit begriffen werden, sondern als »frei verfügbare und gestaltbare Zeit als ein Potential der postfamiliären und nachberuflichen Lebensphase« (Karl 1996: 29). Dieser integrative Ansatz dürfte auch für die Fremdsprachengeragogik leitend sein, betrachtet man die unterschiedlichen Motivationen und damit auch Bedürfnisse der älteren Fremdsprachenlerner, die vom zielgerichteten Lernen einer Fremdsprache und geistigem Training über soziale Motive der Kontaktsuche mit Gleichgesinnten bis hin zu sinnvoller Zeitgestaltung reichen. Ein solcher integrativer Ansatz hat weitreichende Folgen für eine altersgemäße Methodik und Didaktik des Fremdsprachenunterrichts.

Bezogen auf die Lernökologie sind zunächst neue Strukturen der zeitlichen, räumlichen, sozialen und Lerngegenstand-bezogenen Organisation zu entwerfen. Daß das didaktische Setting nicht dem üblichen Schema »Lehrer-Schüler « folgen kann, sondern paritätische Lehr-Lern-Formen einschließen sollte, wird klar, wenn man sich das große Erfahrungswissen älterer Menschen vor Augen hält. Der Biographie-Ansatz, bei dem sich Lernen immer auch auf die individuellen Erfahrungen der Lerner im biographischen Kontext bezieht, ist beim Fremdsprachenlernen besonders dann interessant, wenn Sprachlernerfahrungen und damit auch Methodenerfahrungen und die damit verbundenen Strategieerfahrungen thematisiert werden können. Subjektorientiertheit und Lernerautonomie sind im Rahmen der Fremdsprachengeragogik neu zu definieren. Der Kurs in der Funktion eines Ortes sozialen Miteinanders fordert eine Integration von Kommunikationsebenen, die über das Vermitteln der fremden Sprache hinausweisen und Elemente der Freizeitpädagogik integrieren, um den sozioemotionalen Bedürfnissen älterer Menschen gerecht zu werden.

Abschließend sei angemerkt, daß sich der vorliegende Artikel mit den Spezifika der 
größten Gruppe Fremdsprachen lernender älterer Menschen beschäftigt, die an den Volkshochschulen und ähnlichen Bildungseinrichtungen europaweit anzutreffen sind. Die Zielgruppe älterer Migranten und Aussiedler kann über diese Erhebung nicht erfaßt werden, da sie allein bezogen auf eine Analyse der Motivationen beträchtlich von der vorliegenden Untersuchungsgruppe abweicht (vgl. Kaufmann 1993). Zudem sind ältere Migranten und Aussiedler wenig bildungsgewohnt, ihr Aufenthalt in der Bundesrepublik ist geprägt vom - meist nie realisierten - Wunsch, in das Herkunftsland zurückzukehren, und von einem Rückzug in die Ethnie mit zunehmendem Alter (vgl. Becker 2000). Glaubt man den prognostizierten Zahlen, nach denen sich die Zahl der älteren Aussiedler und Migranten in den nächsten 20 Jahren mehr als verdreifachen werden (Naegele/Olbermann/Gerling 1997: $233 \mathrm{f}$.), sollte auch diese didaktische Zielgruppe stärker ins Blickfeld der Wissenschaft kommen. Becker schreibt hierzu:

»Es ist anzunehmen, daß mit der Zunahme älterer Migranten Bedarf an speziellen Deutschkursen für Ältere entsteht; auch das Bedürfnis, die eigene Muttersprache wieder aufzufrischen, Literatur des Herkunftslandes zu lesen oder überhaupt erst einmal das Schreiben $\mathrm{zu}$ erlernen, wird geäußert.« (Becker 2000: 323)

Auch ältere Fremdsprachenexperten (vgl. Rück 1998) werden sich aufgrund ihres breiten fachspezifischen Wissens in ihren Ansprüchen an Bildungsangebote beträchtlich von den Besuchern von Volkshochschulkursen unterscheiden. Letztendlich kann die beobachtbare Inhomogenität von Lerngruppen Älterer als Konsequenz des Alterns als Prozeß zunehmender Individualisierung mit gleichzeitiger interindividueller Variierung der einzelnen Lerner gesehen werden.

\section{Literatur}

Amaschus, Almut: »Die neuen Alten haben sich noch viel vorgenommen. Reiseverhalten von Senioren - heute und morgen«, FVW international - unabhängige Fachzeitschrift für Touristik und Geschäftsreiseverkehr 20 (1996), $40 \mathrm{f}$.

Baltes, Margret M.; Kohli, Martin; Sames, Karl (Hrsg.): Erfolgreiches Altern. Bedingungen und Variationen. Bern et al.: Hans Huber, 1989.

Baltes, Paul B.: »Entwicklungspsychologie der Lebensspanne: Theoretische Leitsätze«, Psychologische Rundschau 41 (1990), 1-24.

Becker, Susanne: »Ältere Migranten«. In: Becker, Susanne; Veelken, Ludger; Wallraven, Klaus Peter (Hrsg.): Handbuch Altenbildung. Opladen: Leske + Budrich, 2000, 218-325.

Becker, Susanne; Veelken, Ludger; Wallraven, Klaus Peter (Hrsg.): Handbuch Altenbildung. Opladen: Leske + Budrich, 2000.

Berger, Peter; Hradil, Stefan (Hrsg.): Lebenslagen, Lebensläufe, Lebensstile. Göttingen: Schwartz, 1990.

Berndt, Annette: »Fremdsprachenlernen im höheren Erwachsenenalter. Ansätze zu einer Sprachgeragogik «, Info DaF 24, 1 (1997), 69-78.

Brech, Joachim (Hrsg.): Migration - Stadt im Wandel. Darmstadt: Verlag für wissenschaftliche Publikationen, 1997.

Cattell, Raymond B.: »Theory of fluid and crystallized intelligence«, Journal of Educational Psychology 54 (1963), 1-22.

Deutsches Institut für Erwachsenenbildung (Hrsg.): Volkshochschulstatistik. Arbeitsjahr 1997. Frankfurt a. M. 1999.

Freericks, Renate: Was, wenn nicht jetzt? Ziele und Träume für den Ruhestand. Ergebnisse einer Untersuchung. Bielefeld: IFKA, 1990.

Gruner \& Jahr - Märkte und Tendenzen (Hrsg.): Studienreisen. G \& J Marktanalyse. Hamburg 1998.

Jansen, Birgit; Karl, Fred; Radebold, Hartmut; Schmitz-Scherzer, Reinhard (Hrsg.): Soziale Gerontologie. Ein Handbuch für Lehre und Praxis. Weinheim; Basel: Beltz, 1999.

Kaufmann, Susan: »Sprachkurse für ältere Ausländerinnen und Ausländer ", Deutsch lernen 3 (1993), 265-273. 
Kaiser, Mechthild: Bildung durch ein Studium im Alter. Münster et al.: Waxmann, 1997.

Karl, Fred: Performanz im Kontext. Kassel, 1996 (Kasseler gerontologische Schriften 20).

Klercq, Jumbo: »Europäische Erfahrungen«. In: Becker, Susanne; Veelken, Ludger; Wallraven, Klaus Peter (Hrsg.): Handbuch Altenbildung. Opladen: Leske + Budrich, 2000, 140-153.

Kuypers, J. A.; Bengston, V. L.: »Social Breakdown and Competence: A Model of Normal Aging «, Human Development 23 (1973), 181-201.

Lehr, Ursula: Psychologie des Alterns. 7. Auflage. Heidelberg: Quelle \& Meyer, 1991.

Master Class. Sprachurlaub für Menschen ab 50 Jahre. Köln 2000 (Folder der Carl Duisberg Centren).

Mißler, Bettina: Fremdsprachenlernerfahrungen und Lernstrategien. Eine empirische Untersuchung. Tübingen: Stauffenburg, 1999.

Naegele, Gerhard; Olbermann, Elke; Gerling, Vera: »Älter werden in der Fremde «. In: Brech, Joachim (Hrsg.): Migration Stadt im Wandel. Darmstadt: Verlag für wissenschaftliche Publikationen, 1997, 233-234.

Nittel, Dieter: »Erziehungswissenschaften/ Pädagogik «. In: Jansen, Birgit; Karl, Fred; Radebold, Hartmut; Schmitz-Scherzer, Reinhard (Hrsg.): Soziale Gerontologie. Ein Handbuch für Lehre und Praxis. Weinheim; Basel: Beltz, 1999, 356-370.

Olbrich, Erhard; Sames, Klaus; Schramm, Axel (Hrsg.): Kompendium der Gerontologie. Interdisziplinäres Handbuch für Forschung, Klinik und Praxis. Landsberg am Lech: Ecomed, 1994 (Loseblattsammlung).

Oswald, Wolf Dieter: »Psychologische Alter(n)shypothesen «. In: Becker, Susanne; Veelken, Ludger; Wallraven, Klaus Peter. (Hrsg.): Handbuch Altenbildung. Opladen: Leske + Budrich, 2000, 106-117.
Prahl, Hans-Werner / Schroeter, Klaus R.: Soziologie des Alterns. Paderborn et al.: Schöningh, 1996.

Rück, Heribert: »Subjektive Theorie und autodidaktisches Sprachenlernen. PräKognitionen und ihre Überprüfung in einem Lernprozess Russisch«, FluL 27 (1998), 204-221.

Sagebiel, Felicitas: »Geschlechtsspezifisches Lernen: Frauen«. In: Becker, Susanne; Veelken, Ludger; Wallraven, Klaus Peter (Hrsg.): Handbuch Altenbildung. Opladen: Leske + Budrich, 2000, 311-318.

Semmelroth, Britta: »Lernen/Bildung auf Reisen«. In: Becker, Susanne; Veelken, Ludger; Wallraven, Klaus Peter (Hrsg.): Handbuch Altenbildung. Opladen: Leske + Budrich, 2000, 271-278.

Smith, Anderson D.; Earles, Julie L.K.: »Memory changes in normal aging «. In: Blanchard-Fields, Fredda; Hess, Thomas M.: Perspectives on cognitive change in adulthood and aging. New York: The McGraw-Hill Companies, 1996, 192-220.

Veelken, Ludger; Gösken, Eva; Pfaff, Matthias (Hrsg.): Gerontologische Bildungsarbeit - Neue Ansätze und Modelle. Hannover: Vincentz, 1994.

Veelken, Ludger: »Geragogik/Sozialgeragogik - eine Antwort auf neue Herausforderungen an gerontologische Bildungsarbeit, Kultur- und Freizeitarbeit «. In: Veelken, Ludger; Gösken, Eva; Pfaff, Matthias (Hrsg.): Gerontologische Bildungsarbeit Neue Ansätze und Modelle. Hannover: Vincentz, 1994, 13-52.

Wingen, Max; Cornelius, Ivar: »Demografische Veränderungen: Der Weg in eine >greying world « «. In: Olbrich, Erhard; Sames, Klaus; Schramm, Axel (Hrsg.): Kompendium der Gerontologie. Interdisziplinäres Handbuch für Forschung, Klinik und Praxis. Landsberg am Lech: Ecomed, 1994, III-3 (Loseblattsammlung). 